\title{
Características Antropométricas y Somatotipo en Seleccionados Chilenos de Remo
}

\author{
Anthropometric Characteristics and Somatotype in Elite Chilean Rowers
}

Gutiérrez-Leyton, L. ${ }^{\text {; }}$ Zavala-Crichton, J. ${ }^{1}$; Fuentes-Toledo, C. ${ }^{2}$ \& Yáñez-Sepúlveda, R. ${ }^{3}$

GUTIÉRREZ-LEYTON, L.; ZAVALA-CRICHTON, J.; FUENTES-TOLEDO, C. \& YÁÑEZ-SEPÚLVEDA, R. Características

antropométricas y somatotipo en seleccionados chilenos de remo. Int. J. Morphol., 38(1):114-119, 2020.

RESUMEN: El objetivo del estudio fue describir las características antropométricas y somatotipo de los seleccionados chilenos de remo. Se evaluaron a 21 deportistas ( 6 mujeres y 15 hombres) con edades promedio de 18,66 $\pm 2,58$ años para las mujeres y de $22,06 \pm 4,71$ años para los hombres. El estudio se realizó previo al clasificatorio Panamericano Lima 2019, al momento de las evaluaciones, los deportistas se encontraban concentrados en el centro de entrenamiento olímpico (CEO) ubicado en Curauma en la región de Valparaíso en Chile. Las evaluaciones antropométricas se realizaron en base al perfil restringido de 25 variables propuesto por Ross \& Kerr (1991), que permitió fraccionar la masa corporal en 5 componentes (tejidos: piel, residual, óseo, adiposo y muscular). Se midieron también la envergadura, la altura ilioespinal y los pliegues del bíceps y supracrestídeo, todas las medidas se realizaron en base al protocolo de marcaje y evaluación propuesto por ISAK. Se uso el método de Carter y Heath para calcular el somatotipo, describiendo los componentes de endomorfía, mesomorfía y ectomorfía respectivamente. Los resultados mostraron un porcentaje de grasa en hombres de 19,34 $\pm 1,59 \%$ y en mujeres de 27,08 $\pm 3,6 \%$ ( $\mathrm{p}=0,003$ ), la masa muscular de hombres fue de 52,69 $\pm 1,78 \%$ y en mujeres de $45,68 \pm 4,19 \%$ (p=0,003), se apreció un somatotipo mesomorfo balanceado tanto en hombres (1,7-5,3-2,2) como mujeres (2,5-3,7-2,7). Se concluye que los seleccionados chilenos de remo presentan un alto desarrollo de masa muscular y un bajo porcentaje de masa grasa, además de un somatotipo mesomorfo balanceado.

PALABRAS CLAVE: Remo; Somatotipo; Composición Corporal; Antropometría.

\section{INTRODUCCIÓN}

Los parámetros fisiológicos y morfológicos específicos son componentes importantes del rendimiento en muchos deportes. Se ha confirmado que ciertas características físicas como la composición corporal, peso y talla pueden influir significativamente en los resultados deportivos (Duquet et al., 2001). El remo se caracteriza por ser un deporte de potencia y resistencia (Jürimäe et al., 2010; Jensen, 2011; Majumdar, Das, \& Mandal, 2017), este deporte consiste en superar una distancia de 2000 metros oficiales (Boegman \& Dziedzic, 2016; Majumdar et al.), en un intervalo de tiempo que varía entre los 320 y 460 segundos (Bourgois et al., 2014; Majumdar et al.). La competición se desarrolla en diferentes modalidades deportivas, en función del sexo y el peso del atleta, además del tipo de embarcación competitiva (Slater et al., 2014; Boegman \& Dziedzic).

La alta exigencia a nivel muscular y cardiovascular, hace del remo un deporte de los más exigentes a nivel físico y mental. En el alto rendimiento (Bechard et al., 2009), las demandas funcionales y fisiológicas dependen principalmente de las características antropométricas del atleta (Battista et al., 2007; Rakovac et al., 2011). En este sentido el remo es un deporte en donde se destacan las aptitudes de fuerza y resistencia, dos aptitudes físicas relacionadas con la composición corporal (Russell et al. 1998). Ciertos parámetros antropométricos del remero, como la estatura, la cantidad de tejido muscular y adiposo condicionan su potencia aeróbica y anaeróbica, es así como dichas características se transforman en elementos predictores de su propio rendimiento competitivo (Bourgois et al., 2014). Los remeros, especialmente aquellos de peso ligero, mantienen un equilibrio dinámico entre su peso corporal, altura y porcentaje de grasa con el fin de mejorar su rendimiento en la competición (Slater et al., 2005; Fukuda et al., 2017).

Las características antropométricas son parte del conjunto de variables biológicas relacionadas con el rendimiento deportivo (Zuñiga Galavíz s\& de León Fierro, 2007). Es

\footnotetext{
${ }^{1}$ Pedagogía en Educación Física, Facultad de Educación y Ciencias Sociales, Universidad Andres Bello, Viña del Mar, Chile.

${ }^{2}$ Club Deportivo Unión La Calera, La Calera, Valparaiso, Chile.

${ }^{3}$ Grupo IRyS, Escuela de Educación Física, Pontificia Universidad Católica de Valparaíso, Viña del Mar, Chile.
} 
por esto, que durante las últimas dos décadas se han desarrollado nuevas tecnologías para mejorar la evaluación de la composición corporal y se han multiplicado los estudios que intentan identificar la importancia del perfil morfológico ideal en diferentes disciplinas deportivas (Cabral et al., 2011). La evaluación antropométrica es una de las herramientas más utilizadas en la caracterización de deportistas de distintas disciplinas y categorías debido a su accesibilidad y costos. Se ha demostrado que durante la etapa de especialización deportiva, los deportistas adquieren algunas características antropométricas diferenciales, en las cuales influyen factores sociales, hereditarios, nutritivos y de entrenamiento, entre otros (Reilly, 2008). Por lo que, mediciones precisas y fiables de su composición corporal proporcionarán información importante para entrenadores a la hora de monitorizar los programas de entrenamiento y nutrición (Slater et al., 2014).

En base a lo señalado, la presente investigación tuvo por objetivo describir las características antropométricas y somatotipo de seleccionados chilenos de remo.

\section{MATERIAL Y MÉTODO}

Muestra: Estuvo constituida por 21 remeros chilenos elite, distribuidos en 6 mujeres (18,66 $\pm 2,58$ años) y 16 hombres ( 22,06 $\pm 4,71$ años). Esta investigación se realizó considerando las recomendaciones de la declaración de Helsinki para estudios con seres humanos. Al momento de las mediciones, todos los atletas se encontraban sanos, sin antecedentes de enfermedades crónicas o agudas.

Materiales: Para la recolección de medidas básicas, se utilizó una balanza digital TANITA ® (Japón) con precisión de $100 \mathrm{~g}$. La talla corporal sentado y de pie, fue medida con un estadiómetro marca SECA (Hamburgo, Alemania), dichas evaluaciones fueron realizadas a pies descalzos. Los diámetros óseos fueron medidos con un antropómetro corto y uno largo del kit de antropometría Health \& Performance®, en la medición de los perímetros se utilizó una cinta métrica metálica marca CESCORF® (Sao Paulo, Brasil) con una resolución de medida: $\pm 1 \mathrm{~mm}$. Finalmente para la toma de los pliegues cutáneos se utilizó un plicómetro científico de calibración manual marca AVANUTRI (Rio de Janeiro, Brasil) con una sensibilidad de $0,1 \mathrm{~mm}$ y una presión $\pm 10 \mathrm{~g} / \mathrm{mm}^{2}$.

Evaluaciones: Las evaluaciones fueron realizadas en el centro de alto rendimiento (CEO) ubicado en Curauma, Valparaíso, Chile.
Las variables evaluadas fueron mayormente las que conforman el perfil restringido propuesto por la International Society for the Advancement of Kineanthropometry (ISAK) (Marfell-Jones et al., 2006). Dichas medidas fueron realizadas por profesionales con certificación ISAK nivel II y III. Todas las evaluaciones se realizaron previo consentimiento informado, donde se señala al deportista acerca de los alcances del estudio, su metodología y los objetivos de la investigación.

Para la evaluación, se dividió al grupo entre mujeres y hombres. Se evaluó un total de 29 variables antropométricas, donde encontramos como medidas básicas (5): peso corporal $(\mathrm{kg})$, talla sentado $(\mathrm{cm})$, talla de pie $(\mathrm{cm})$, envergadura $(\mathrm{cm})$ y altura ilioespinal $(\mathrm{cm})$. En lo que respecta a los diámetros óseos, fueron evaluados (6): biacromial, tórax transverso, tórax anteroposterior, biiliocrestídeo, humeral y femoral. Se midieron perímetros (10): cabeza, brazo relajado, brazo flexionado en tensión, antebrazo, tórax mesoesternal, cintura, cadera, muslo máximo, muslo medial y pantorrilla. Finalizando con la toma de datos se procede a evaluar los pliegues cutáneos (8): bíceps, tríceps, subescapular, suprailiaco, supraespinal, abdominal, muslo frontal y pierna medial. En lo que respecta a la determinación de los 5 componentes del fraccionamiento de la masa corporal (piel, músculo, grasa, óseo y residual), se utilizó el método propuesto por Kerr y Ross (Keer, 1988; Ross \& Kerr); dicho análisis fue interpretado por el programa Excel de Microsoft Office. Se realizó el cálculo de los valores $\mathrm{Z}$ según el modelo de proporcionalidad del Phantom de Ross \& Marfell-Jones. El análisis y distribución del somatotipo se efectuó a través del método propuesto por Heath y Carter (Carter \& Heath, 1990; Carter, 2002). Finalmente para calcular el índice músculo/óseo se dividió el tejido muscular con el tejido óseo en kilogramos.

Análisis estadístico: Para la descripción de las variables se utilizaron los estadísticos media y desviación estándar, los que fueron tabulados y calculados con los programas Microsoft Office Excel versión 2013® (Albuquerque, Estados Unidos) y Graphpad Prism versión 7.0® (California, Estados Unidos) respectivamente. El somatotipo fue calculado con el software Somatotype Calculation and Analysis Software versión 1.1 (San Diego, CA, EE.UU). El análisis de fiabilidad se realizó con el software SPSS versión 25.0 para MAC®. Para comparar los resultados según sexo se utilizó la prueba T de muestras independientes y se consideró un valor $\mathrm{p}<0,05$. Se realizó un análisis de fiabilidad para validar el método en la población considerando el peso estructurado y el peso real evaluado, los cuales muestran un coeficiente de correlación intraclase que fue de 0,989 ( $p<0,001)$, la prueba de correlación de Pearson fue de 0,988 ( $p<0,001)$ y el R2 fue de 0,976 ( $p<0,001)$, lo que muestra una alta fiabilidad, confiabilidad y validez interna del método en la población deportista evaluada. 


\section{RESULTADOS}

Considerando la distribución de todos los componentes estudiados y representados en las siguientes tabulaciones y figuras, se representan los valores resultantes en cada variable, para finalmente describir las principales características morfológicas resumidas en somatocartas separadas según sexo.

Tabla I. Medidas básicas, diámetros, perímetros y pliégues en grupo de remeros evaluados diferenciados por género $(n=21)$.

\begin{tabular}{|c|c|c|c|}
\hline Variables & Mujeres $(n=6)$ & Hombres $(n=15)$ & Valor $\mathrm{p}$ \\
\hline \multicolumn{4}{|l|}{ Medidas Básicas } \\
\hline Edad & $18,66 \pm 2,58$ & $22,06 \pm 4,71$ & 0,050 \\
\hline Peso (kg) & $62,93 \pm 5,64 *$ & $81,56 \pm 7,63 *$ & 0,000 \\
\hline Talla $(\mathrm{cm})$ & $170 \pm 4,61 *$ & $182,02 \pm 4,42 *$ & 0,000 \\
\hline Altura sentado $(\mathrm{cm})$ & $87,88 \pm 2,49 *$ & $92,84 \pm 2,22 *$ & 0,003 \\
\hline Envergadura (cm) & $172,88 \pm 4,90 *$ & $188,66 \pm 5,06 *$ & 0,000 \\
\hline \multicolumn{4}{|l|}{ Diámetros $(\mathrm{cm})$} \\
\hline Altura ilioespinal & $96,68 \pm 3,25 *$ & $104,16 \pm 3,43 *$ & 0,001 \\
\hline Biacromial & $37,2 \pm 1,12 *$ & $41,62 \pm 1,36 *$ & 0,000 \\
\hline Torax Transverso & $25,05 \pm 1,44 *$ & $29,08 \pm 1,30^{*}$ & 0,000 \\
\hline Tor. Anteroposterior & $17,03 \pm 0,93 *$ & $19,67 \pm 1,13 *$ & 0,000 \\
\hline Bi-iliocrestídeo & $25,55 \pm 0,81 *$ & $27,26 \pm 1,42 *$ & 0,003 \\
\hline Humeral & $6,5 \pm 0,18 *$ & $7,34 \pm 0,34 *$ & 0,000 \\
\hline Femoral & $9,15 \pm 0,49 *$ & $10,27 \pm 0,57 *$ & 0,001 \\
\hline \multicolumn{4}{|l|}{ Perímetros $(\mathrm{cm})$} \\
\hline Cabeza & $54,55 \pm 0,64 *$ & $56,9 \pm 0,83 *$ & 0,000 \\
\hline Brazo Relajado & $28,1 \pm 1,72 *$ & $32,55 \pm 2,09 *$ & 0,000 \\
\hline Brazo Flex. Máx & $28,28 \pm 1,49 *$ & $34,49 \pm 1,99 *$ & 0,000 \\
\hline Antebrazo & $23,76 \pm 1,21 *$ & $28,64 \pm 1,29 *$ & 0,000 \\
\hline Torax Mesoesternal & $86,11 \pm 4,06 *$ & $100,44 \pm 5,07 *$ & 0,000 \\
\hline Cintura Mínima & $67,75 \pm 1,47 *$ & $80,02 \pm 3,06 *$ & 0,000 \\
\hline Cadera Máx & $91,76 \pm 4,14 *$ & $97,46 \pm 4,80 *$ & 0,021 \\
\hline Muslo Máx & $54,41 \pm 3,77 *$ & $58,93 \pm 3,56 *$ & 0,034 \\
\hline Muslo Medio & $50,85 \pm 3,29 *$ & $55,76 \pm 3,05 *$ & 0,012 \\
\hline Pantorrilla Max & $33,36 \pm 2,54 *$ & $36,84 \pm 1,97 *$ & 0,018 \\
\hline \multicolumn{4}{|c|}{ Pliegues Cutáneos (mm) } \\
\hline Bíceps & $3,5 \pm 1,3$ & $2,46 \pm 0,42$ & 0,162 \\
\hline Tríceps & $10,55 \pm 5,18$ & $6,89 \pm 1,58$ & 0,147 \\
\hline Subescapular & $8,03 \pm 1,79$ & $6,84 \pm 1,36$ & 0,186 \\
\hline Supracrestideo & $8,76 \pm 2,25$ & $10,04 \pm 3,74$ & 0,354 \\
\hline Supraespinal & $6,33 \pm 1,97$ & $5,4 \pm 2,04$ & 0,357 \\
\hline Abdomin al & $10,51 \pm 2,79$ & $10,12 \pm 3,50$ & 0,790 \\
\hline Muslo Medial & $13,23 \pm 5,22$ & $8,38 \pm 2,23$ & 0,073 \\
\hline Pantorrilla & $9,46 \pm 5,30^{*}$ & $4,12 \pm 1,31^{*}$ & 0,008 \\
\hline \multicolumn{4}{|c|}{ Sumatoria de pliegues $(\mathrm{mm})$} \\
\hline$\Sigma 6$ Pliegues & $58,13 \pm 16,81^{*}$ & $42,36 \pm 10,19 *$ & 0,016 \\
\hline$\Sigma 8$ Pliegues & $70,30 \pm 16,80^{*}$ & $54,87 \pm 13,76^{*}$ & 0,042 \\
\hline \multirow{2}{*}{\multicolumn{4}{|c|}{$\begin{array}{l}\text { Índice mús culo óseo } \\
\text { (músculo }{ }^{\mathrm{kg}} / \mathrm{huesokg} \text { ) }\end{array}$}} \\
\hline & & & \\
\hline IMO & $4,09 \pm 0,57^{*}$ & $4,68 \pm 0,47 *$ & 0,027 \\
\hline
\end{tabular}

*Diferencias significativas considerando $\mathrm{p}<0,05$.
La Tabla I, resume los resultados obtenidos para las medidas básicas, diámetros, perímetros, pliegues, sumatoria de pliegues e índice músculo óseo (IMO) de ambos sexos.

En la Tabla II se presentan los resultados diferenciados por sexo de los valores medios y desviación estándar de los cinco componentes correspondiente al método pentacompartimental, categorizados en kilogramos, porcentaje y su respectivo Phantom Z.

En la Tabla III se exponen los componentes resultantes para el somatotipo de los remeros, diferenciados por sexo y su posterior clasificación. Obteniendo diferencias significativas en el compomente Endomorfía y Mesomorfía, clasificando a ambos sexos en la categoría de mesomorfo balanceado, un cuerpo con un desarrollo de masa muscular elevado y con un porcentaje graso bajo que recubre los contornos corporales.

En la Figura 1 se aprecia la somatocarta obtenida para las mujeres pertenecientes a la selección nacional de remo, en esta se observa un somatotipo promedio con valores 2,5-3,7-2,7, el que se clasificó como mesomorfo balanceado.

A su vez, en la Figura 2, también se resumen los valores obtenidos para las somatocartas de los hombres, el cual en sus valores promedio de 1,7-5,3-2,2, el cual los categoriza en mesomorfo balanceado.

\section{DISCUSIÓN}

Los resultados de la presente investigación evidencian, que los hombres presentan un peso mucho mayor (hombres: $81,56 \pm 7,63 \mathrm{~kg} /$ mujeres: $62,93 \pm 5,64$ $\mathrm{kg}$ ), lo mismo ocurre con la talla (hombres: $182,02 \pm 4,42 \mathrm{~cm} /$ mujeres: $170 \pm 4,61 \mathrm{~cm})$ y altura sentado (hombres: $92,84 \pm 2,22 \mathrm{~cm}$ / mujeres: $87,88 \pm 2,49 \mathrm{~cm})$. Tras una revi- 
Tabla II. Tejido Adiposo, muscular, residual, óseo, piel y componentes endomorfía, mesomorfía y ectomorfía del grupo de remeros elite evaluadios.

\begin{tabular}{|c|c|c|c|c|}
\hline Variable & & Mujeres & Hombres & Valor $\mathrm{p}$ \\
\hline \multicolumn{5}{|c|}{ Tejido Adiposo } \\
\hline & $(\%)$ & $27,08 \pm 3,64 *$ & $19,34 \pm 1,59 *$ & 0,003 \\
\hline & $(\mathrm{Kg})$ & $16,96 \pm 2,01$ & $15,82 \pm 2,30$ & 0,288 \\
\hline \multicolumn{5}{|c|}{ Tejido Muscular } \\
\hline & $(\%)$ & $45,68 \pm 4,19 *$ & $52,69 \pm 1,78 *$ & 0,008 \\
\hline & $(\mathrm{Kg})$ & $28,89 \pm 4,86 *$ & $43,02 \pm 4,78 *$ & 0,000 \\
\hline \multicolumn{5}{|c|}{ Tejido Óseo } \\
\hline & $(\%)$ & $11,23 \pm 0,69$ & $11,33 \pm 0,93$ & 0,788 \\
\hline & $(\mathrm{Kg})$ & $7,04 \pm 0,48 *$ & $9,20 \pm 0,62 *$ & 0,000 \\
\hline \multicolumn{5}{|c|}{ Tejido Residual } \\
\hline & $(\%)$ & $9,92 \pm 1,12 *$ & $11,36 \pm 0,53 *$ & 0,025 \\
\hline & $(\mathrm{Kg})$ & $6,11 \pm 0,46 *$ & $9,24 \pm 0,66 *$ & 0,000 \\
\hline \multicolumn{5}{|c|}{ Tejido Piel } \\
\hline & $(\%)$ & $6,25 \pm 0,43 *$ & $5,25 \pm 0,30 *$ & 0,001 \\
\hline & $(\mathrm{Kg})$ & $3,91 \pm 0,20 *$ & $4,26 \pm 0,22 *$ & 0,007 \\
\hline
\end{tabular}

*Diferencias significativas considerando $\mathrm{p}<0,05$.

Tabla III.- Somatotipo de los deportistas evaluados según sexo y su clasificación resultante.

\begin{tabular}{lccc}
\hline Variable & Mujeres & Hombres & Valor p \\
\hline Endomorfía & $2,5 \pm 0,72^{*}$ & $1,7 \pm 0,49^{*}$ & 0,006 \\
Mesomorfía & $3,7 \pm 1,03^{*}$ & $5,3 \pm 0,81^{*}$ & 0,001 \\
Ectomorfía & $2,7 \pm 0,96$ & $2,2 \pm 0,62$ & 0,136 \\
Clasificación del somatotipo & Mesomorfo Balanceado & Mesomorfo Balanceado &
\end{tabular}

*Diferencias significativas considerando $\mathrm{p}<0,05$.

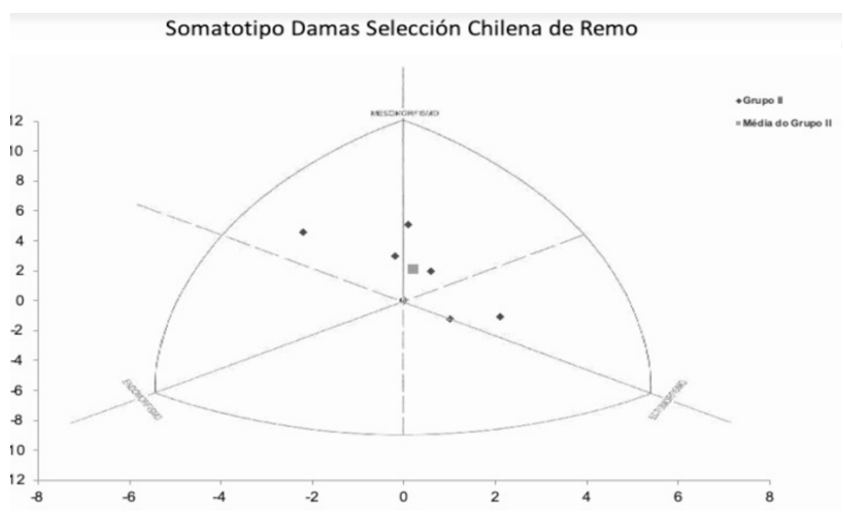

Fig. 1. Somatotipo en el grupo de mujeres $(n=6)$.

sión de la literatura, diversas investigaciones dan cuenta de la importancia de la composición corporal como una determinante del rendimiento deportivo. Akça (2014) concluyó que la altura del cuerpo $($ Media $=185,77 \mathrm{~cm}$ ) y la masa corporal $($ Media $=80,23 \mathrm{~kg}$ ) se correlacionaban significativamente con el rendimiento en remo. En la misma línea, Mikulic (2009) concluyó en su artículo, que los remeros de mayor éxito internacional son más altos (191,0

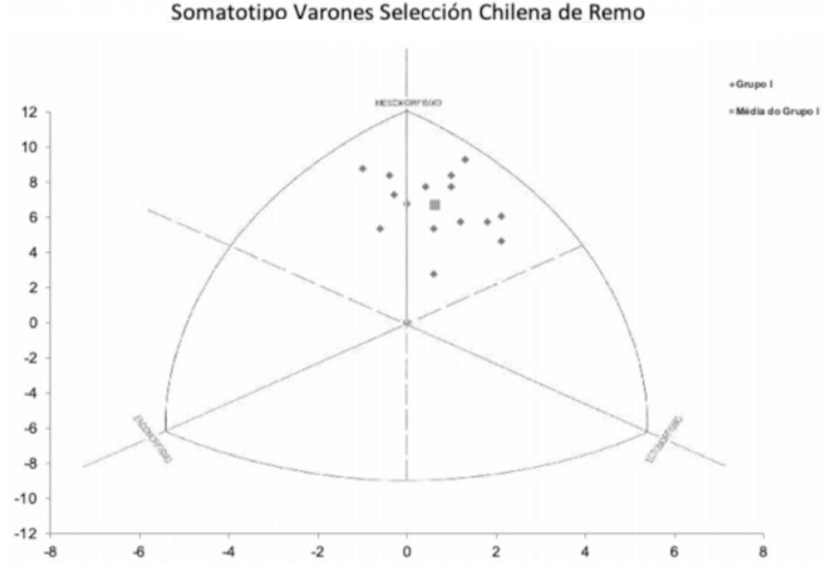

Fig. 2. Somatotipo en el grupo de hombres $(n=15)$.

$\pm 0,05 \mathrm{~cm})$ y pesados $(91,7 \pm 5,9 \mathrm{~kg})$, con una mayor altura sentados y con una masa grasa inferior $(13,2 \pm 2,3 \%)$ a aquellos remeros que presentaban menor rendimiento. Otro estudio similar realizado en mujeres por Podstawski et al. (2012), concluyen que la altura $(171,2 \pm 7,67 \mathrm{~cm})$ y el peso $(63,98 \pm 10,21 \mathrm{~kg})$ estaban relacionados con el tiempo requerido para realizar $1000 \mathrm{~m}$ en remo, tanto en remeros de élite como en personas físicamente inactivas. 
Los estudios antropométricos en remeros se centran en su mayoría en la importancia de la altura y tamaño de la masa corporal como un factor determinante del rendimiento en remo. Gran parte de las investigaciones centran su mirada en la altura y el tamaño de la masa corporal del deportista, sin embargo, las miembros especialmente largos, que acompañan al remero de gran estatura, favorecen un mayor recorrido de palada y un aumento de la potencia de impulso de ésta (Claessens et al., 2005). El éxito deportivo en el remo, se ve aventajado por poseer miembros inferiores y superiores más largos, influyendo positivamente en la propulsión de la embarcación (Rakovac et al.).

Guereño y colaboradores en el año 2018 sugieren que la envergadura $(188,6 \pm 5,8)$, parece ser de gran importancia para los remeros de elite, mientras que la altura promedio $(182,5 \pm 5,2)$ puede no ser tan imporante para el rendimiento. Para el caso de la presente investigación, mujeres y hombres obtuvieron valores significativamente mayores en relación a la envergadura (mujeres: $172,88 \pm 4,90 \mathrm{~cm} /$ hombres: $188,66 \pm 5,06 \mathrm{~cm}$ ) en comparación con la talla (mujeres: $170 \pm 4,61 \mathrm{~cm} /$ hombres: $182,02 \pm 4,42 \mathrm{~cm}$ ), por lo cual su rendimiento se ve favorecido debido a la hipótesis señalada por Guereño.

En cuanto al somatotipo promedio obtenido en el presente estudio, éste se clasifica como mesomórfico balanceado para mujeres $(2,5-3,7-2,7)$ y hombres $(1,7-5,3-2,2)$ respectivamente.

Los porcentajes de tejido adiposo en mujeres dieron como resultado $27,08 \pm 3,64 \%(16,96 \pm 2,01 \mathrm{~kg})$, mientras que la masa muscular obtenida fue de 45,68 $\pm 4,19 \%(28,89$ $\pm 4,86 \mathrm{~kg}$ ). Por otra parte, los hombres obtuvieron un porcentaje de tejido adiposo de 19,34 $\pm 1,59 \%(15,82 \pm 2,30$ $\mathrm{kg})$ y una masa muscular de $52,69 \pm 1,78 \%(43,02 \pm 4,78$ $\mathrm{kg})$.

En Chile existe solo un estudio que trató la misma temática en hombres $(n=27)$ categoría juvenil $(16,95 \pm 0,97$ edad), utilizando el mismo criterio de evaluación (método pentacompartimental), obteniendo un somatotipo mesomórfico balanceado (2,62 - 4,75 - 2,41) (Gajardo et al, 2013). Sin embargo, dicho estudio contemplaba solo a categorías juveniles y no se consideraba a mujeres dentro de su investigación. Recientes estudios realizados en remeros de élite (Guereño et al, 2018), utilizando diferentes fórmulas para determinar el porcentaje graso, obtuvieron como resultados los siguientes valores: Fórmula de Carter 8,0 \% $( \pm 1,2)$; Withers $9,9 \%( \pm 2,0)$; Yuhasz $8,6 \%( \pm 1,1)$ y Faulkner $10,9 \%( \pm 1,1)$. Respecto a la masa muscular, esta fue de $43,3 \%( \pm 2,4)$ según la ecuación Lee y el somatotipo obtenido fue de $3,5( \pm 0,4)-$ $4,7( \pm 0,6)-2,4( \pm 0,6)$, clasificando a dichos deportistas con un somatotipo endo-mesomorfo. Es importante conocer como se conforma la masa corporal de un remero de élite, es así como Izquierdo-Gabarren et al. (2010) en su investigación concluyen que los remeros que poseen un porcentaje graso bajo $(12,3 \pm 1 \%)$ versus un gran desarrollo de masa muscular $(73,7 \pm 4 \mathrm{~kg})$ tienen una mayor probabilidad de triunfar. En el caso de los remeros categoría ligeros, también alcanzan un mejor rendimineto aquellos que poseen un menor porcentaje de grasa corporal (mujeres: 7,1 \pm 1,3 / hombres: 5,2 $\pm 0,7 \%$ ) y mayor masa muscular total (mujeres: 25,5 $\pm 1,3 \%$ / hombres: 34,9 $\pm 1,2$ ) (Slater et al., 2005).

\section{CONCLUSIÓN}

Los resultados obtenidos muestran un gran desarrollo de la masa muscular, un bajo tejido graso y una gran envergadura, características positivas para el rendimiento de remeros elite chilenos. El somatotipo con clasificación mesomorfo balanceado en ambos sexos describe y orienta la composición y distribución corporal que presentan tanto hombres como mujeres. Los hombres presentan mayor desarrollo muscular y menor cantidad de tejido adiposo que las mujeres. Este artículo aporta la descripción de los remeros élite chilenos en ambos sexo, la cual puede ser utilizada para guiar los procesos de planificación, nutrición y monitorización del entrenamiento.

GUTIÉRREZ-LEYTON, L.; ZAVALA-CRICHTON, J.; FUENTES-TOLEDO, C. \& YÁÑEZ-SEPÚLVEDA, R. Anthropometric characteristics and somatotype in elite Chilean rowers. Int. J. Morphol., 38(1):114-119, 2020.

SUMMARY: The aim of the study was to describe the anthropometric characteristics and somatotype of the Chilean selected rowers. Twenty one 21 athletes ( 6 women and 15 men) with an average age of $18.66 \pm 2.58$ years for women and $22.06 \pm$ 4.71 years for men were evaluated. The study was conducted prior to the Pan American qualification in Lima 2019. At the time of the evaluations, the athletes were concentrated in the Olympic Training Center (CEO) located in Curauma in the Valparaíso region of Chile. The anthropometric evaluations were carried out based on the restricted profile of 25 variables proposed by Kerr and Ross that allowed the fractionation of the body mass into 5 components (tissue: skin, residual, bone, adipose and muscle). The wingspan, the iliospinal height and the folds of the biceps and supracrestide were also measured; all measurements were based on the protocol of marking and evaluation proposed by ISAK. Carter and Heath method was used to calculate the somatotype, describing the components of endomorphy, mesomorphy and ectomorphy respectively. The results showed a percentage of fat in men of 19.34 $\pm 1.59 \%$ and in women of $27.08 \pm 3.6 \%(\mathrm{p}=0.003)$, the muscle mass of men was $52.69 \pm 1.78 \%$ and in women of $45.68 \pm 4.19 \%$ 
$(\mathrm{p}=0.003)$, a balanced mesomorphic somatotype was observed in both men (1.7-5.3-2.2) and women (2.5-3, 7-2.7). It is concluded that the Chilean rowing selected have a high development of muscle mass and a low percentage of fat mass, in addition to a balanced mesomorphic somatotype.

KEY WORDS: Rowing; Somatotype; Body Composition; Anthropometry.

\section{REFERENCIAS BIBLIOGRÁFICAS}

Akça, F. Prediction of rowing ergometer performance from functional anaerobic power, strength and anthropometric components. J. Hum. Kinet., 41:133-42, 2014.

Battista, R. A.; Pivarnik, J. M.; Dummer, G. M.; Sauer, N. \& Malina, R. M. Comparisons of physical characteristics and performances among female collegiate rowers. J. Sports Sci., 25(6):651-7, 2007.

Bechard, D. J.; Nolte, V.; Kedgley, A. E. \& Jenkyn, T. R. Total kinetic energy production of body segments is different between racing and training paces in elite Olympic rowers. Sports Biomech., 8(3):199211, 2009.

Boegman, S. \& Dziedzic, C. E. Nutrition and supplements for elite openweight rowing. Curr. Sports Med. Rep., 15(4):252-61, 2016.

Bourgois, J.; Steyaert, A. \& Boone, J. Physiological and anthropometric progression in an international oarsman: a 15-year case study. Int. $J$. Sports Physiol. Perform., 9(4):723-6, 2014.

Cabral, B. G. A. T.; Cabral, S. A. T.; de Miranda, H. F.; Dantas, P. M. S. \& Reis, V. M. Discriminant effect of morphology and range of attack on the performance level of volleyball players. Rev. Bras. Cineantropom. Desempenho Hum., 13(3):223-9, 2011.

Carter, J. E. L. \& Heath, B. H. Somatotyping. Development and Applications. Cambridge, Cambridge University Press, 1990. pp.398420.

Carter, J. E. L. The Heath-Carter Anthropometric Somatotype. Instruction Manual. San Diego, Department of Exercise and Nutritional Sciences, San Diego State University, 2002.

Claessens, A. L.; Bourgois, J.; Van Aken, K.; Van der Auwera, R.; Philippaerts, R.; Thomis, M.; Vrijens, J.; Loos, R. \& Lefevre, J. Body proportions of elite male junior rowers in relation to competition level, rowing style and boat type. Kinesiology, 37(2):123-32, 2005.

Duquet, W.; Carter, J.; In, S.; Eston, R. \& Reilly, T. Somatotyping. In: Eston, R. \& Reilly, T. (Eds.). Kinanthropometry and Exercise Physiology Laboratory Manual. Anthropometry. $2^{\text {nd }}$ ed. London, Routledge, 2001. pp.47-64.

Fukuda, D. H.; Wray, M. E.; Kendall, K. L.; Smith-Ryan, A. E. \& Stout, J. R. Validity of near-infrared interactance (FUTREX 6100/XL) for estimating body fat percentage in elite rowers. Clin. Physiol. Funct. Imaging, 37(4):456-8, 2017.

Izquierdo-Gabarren, M.; Expósito, R. G.; de Villarreal, E. S. \& Izquierdo, M. Physiological factors to predict on traditional rowing performance. Eur. J. Appl. Physiol., 108(1):83-92, 2010.

Jensen, A. M. The use of Neuro Emotional Technique with competitive rowers: A case series. J. Chiropr. Med., 10(2):111-7, 2011.

Jürimäe, T.; Perez-Turpin, J. A.; Cortell-Tormo, J. M.; Chinchilla-Mira, I. J.; Cejuela-Anta, R.; Mäestu, J.; Purge, P. \& Jürimäe, J. Relationship between rowing ergometer performance and physiological responses to upper and lower body exercises in rowers. J. Sci. Med. Sport, 13(4):434-7, 2010

Kerr, D. An Anthropometric Method for Fractionation of Skin, Adipose, Bone, Muscle and Residual Masses in Males and Females Age 6 to 77 Years. M. Sc. Kinesiology Thessis. British Columbia, Simon Fraser University, 1988.
Majumdar, P.; Das, A. \& Mandal, M. Physical and strength variables as a predictor of $2000 \mathrm{~m}$ rowing ergometer performance in elite rowers. $J$. Phys. Educ. Sport, 17(4):2502-7, 2017.

Marfell-Jones, M.; Stewart, A. \& Carter, J. International Standards for Anthropometric Assessment. Sydney, UNSW Press, 2006.

Mikulic, P. Anthropometric and metabolic determinants of 6,000-m rowing ergometer performance in internationally competitive rowers. J. Strength Cond. Res., 23(6):1851-7, 2009.

Podstawski, R.; Choszcz, D.; Siemianowska, E. \& Skibniewska, K. A. Determining the effect of selected anthropometric parameters on the time needed to cover 1000 m on a rowing ergometer by physically inactive young women. Isokinet. Exerc. Sci., 20(3):197-204, 2012.

Rakovac, M.; Smoljanovic, T.; Bojanic, I.; Hannafin, J. A.; Hren, D. \& Thomas, P. Body size changes in elite junior rowers: 1997 to 2007. Coll. Antropol., 35(1):127-31, 2011.

Reilly, T. The international face of sports science through the window of the Journal of Sports Sciences--with a special reference to kinanthropometry. J. Sports Sci., 26(4):349-63, 2008.

Ross, W. D. \& Kerr, D. A. Fraccionamiento de la masa corporal: Un nuevo método para utilizar en nutrición clínica y medicina deportiva. Apunts Med. Esport, 28(109):175-88, 1991.

Russell, A. P.; Le Rossignol, P. F. \& Sparrow, W. A. Prediction of elite schoolboy $2000 \mathrm{~m}$ rowing ergometer performance from metabolic, anthropometric and strength variables. J. Sports Sci., 16(8):749-54, 1998.

Slater, G. J.; Rice, A. J.; Mujika, I.; Hahn, A. G.; Sharpe, K. \& Jenkins, D. G. Physique traits of lightweight rowers and their relationship to competitive success. Br. J. Sports Med., 39(10):736-41, 2005.

Slater, G.; Rice, A.; Jenkins, D. \& Hahn, A. Body mass management of lightweight rowers: nutritional strategies and performance implications. Br. J. Sports Med., 48(21):1529-33, 2014.

Zuñiga Galavíz, U. \& de León Fierro, L. G. Somatotipo en futbolistas semiprofesionales clasificados por su posición de juego. Rev. Int. Cienc. Deporte, 3(9):29-36, 2007.

\author{
Dirección para correspondencia: \\ Luis Gutiérrez-Leyton. \\ Universidad Andrés Bello \\ Dirección: Av. Los Castaños \#404 \\ Viña del Mar \\ Valparaíso \\ CHILE
}

Email: luis.gutierrez@unab.cl

Recibida : 08-07-2019

Aceptada: 05-08-2019 